\title{
Artigo/Article
}

\section{Estudo dos efeitos adversos e do efeito protetor da vacina contra influenza em idosos vacinados pela rede pública no município de Tubarão, Estado de Santa Catarina}

\author{
Study on the adverse effects and protective effects of influenza vaccine among elderly individuals \\ vaccinated through the public system in the municipality of Tubarão, State of Santa Catarina
}

\author{
Talita Siemann Santos Pereira ${ }^{1}$, Aracelli Tavares Freire ${ }^{1}$, Alixandre Dias Braga ${ }^{1}$, Gregório Wrublevski Pereira ${ }^{1}$, \\ Carine Raquel Blatt ${ }^{2}$ e Alessandra Abel Borges ${ }^{3}$
}

\begin{abstract}
RESUMO
Introdução: $\mathrm{O}$ presente estudo buscou identificar os efeitos adversos e o efeito protetor da vacina contra influenza, em idosos acima de 60 anos, aplicada pela rede pública no município de Tubarão, Santa Catarina. Métodos: Foi realizada uma coorte prospectiva de pacientes durante o período de maio a setembro de 2008. Foram recrutados 341 idosos, sendo 289 vacinados na rede pública (VSUS) e 52 não vacinados (NV). A incidência do efeito protetor foi verificada através da comparação dos grupos VSUS com os NV. Resultados: Um percentual de $22,5 \%$ de pacientes apresentou pelo menos um efeito adverso. Comparando o grupo VSUS e NV quanto à incidência de sintomas gripais durante o inverno, a presença de pelo menos um sintoma foi verificada em $47 \%$ e $28,8 \%(\mathrm{RR}=1,11 ; 1,02-1,22 ; \mathrm{p}=0,0156)$, respectivamente. Conclusões: Os resultados apontam uma baixa incidência de efeitos adversos. A maior incidência de sintomas gripais no grupo VSUS comparado ao NV pode estar relacionado às características da população que costuma fazer a vacina contra influenza.
\end{abstract}

Palavras-chaves: Influenza humana. Vacinas. Efeitos adversos. Infecções respiratórias.

\begin{abstract}
Introduction: The aim of this study was to investigate the incidence of adverse effects and protective effects from influenza vaccine among elderly individuals who were vaccinated through the public healthcare system in the municipality of Tubarão, State of Santa Catarina. Methods: A prospective cohort study was conducted between May and September 2008. A group of 341 patients was recruited, among whom 289 had been vaccinated through the public system (VSUS) and 52 had not been vaccinated (NV). The incidence of protective effects was observed by comparing the VSUS and NV groups. Results: It was observed that $22.5 \%$ of the vaccinated subjects exhibited at least one adverse effect. Comparing the VSUS and NV groups in relation to the incidence of flu symptoms presented during the winter, at least one symptom was observed in $47 \%$ and $28.8 \%(\mathrm{RR}=1.11 ; 1.02-1.22 ; \mathrm{p}=0.0156)$, respectively. Conclusions: These findings demonstrate that the incidence of adverse effects was low. The high incidence of flu symptoms in the VSUS group, in comparison with NV, may be related to the profile of subjects who are in the habit of getting vaccinated against influenza.
\end{abstract}

Keywords: Human influenza. Vaccines. Adverse effects. Respiratory tract infections.

1. Curso de Medicina, Universidade do Sul de Santa Catarina, Tubarão, SC. 2. Curso de Farmácia, Universidade do Sul de Santa Catarina, Tubarão, SC 3. Setor de Imunologia e Virologia, Instituto de Ciências Biológicas e da Saúde, Universidade Federal de Alagoas, Maceió, AL.

Endereço para correspondência: Dr ${ }^{2}$ Alessandra Abel Borges. ICBS/UFAL. Praça Afrânio Jorge s/n, Prado, 57010-020 Maceió, AL.

Tel: 5582 3336-3444

e-mail: alessandra.a.borges@gmail.com

Recebido para publicação em 15/07/2010

Aceito em $06 / 10 / 2010$

\section{INTRODUÇÃO}

A influenza é doença infecciosa aguda de origem viral que acomete o trato respiratório. A cada inverno, a doença atinge mais de 100 milhões de pessoas na Europa, Japão e Estados Unidos, causando, anualmente, a morte de cerca de 20 a 40 mil pessoas somente neste último país ${ }^{1}$. Estima-se que, a cada ano, cerca de $10 \%$ da população mundial apresenta um episódio de influenza ${ }^{2}$.

As vacinas continuam a se constituir nos procedimentos de melhor relação custo/efetividade no setor saúde ${ }^{3}$. O declínio acelerado da morbimortalidade por doenças imunopreveníveis nas décadas recentes, em nosso país e em escala mundial, serve de prova inconteste do enorme benefício que é oferecido às populações através das vacinas $^{3}$. Desde 1999, o Ministério da Saúde (MS) institui as campanhas de vacinação contra influenza para a população acima de $60 \operatorname{anos}^{4}$. O objetivo dessas campanhas é a redução da mortalidade e morbidade relacionadas à doença e, consequentemente, a melhoria da qualidade de vida desta população ${ }^{1}$.

Muitos estudos têm demonstrado o impacto da vacinação contra a influenza na prevenção de internações e mortes por pneumonias e outras doenças, tanto em idosos saudáveis como em populações de risco, particularmente em períodos de maior circulação do vírus ${ }^{2,5-9}$. Contudo, outros estudos demonstraram resultados inesperados, questionando a eficácia da vacina em idosos ${ }^{10,11}$ e na incidência de morte ou pneumonia em adultos ${ }^{12}$.

Além disso, no Brasil, há poucos trabalhos publicados que apresentam dados a respeito de reações adversas da vacina contra influenza ${ }^{13,14}$. Donalísio cols ${ }^{15}$ apontaram em seu estudo que é frequente a subnotificação e a falta de sistematização destes dados pelo Sistema de Vigilância Epidemiológica.

A preocupação com a ocorrência de eventos adversos tem sido apontada como um fator que 
contribui para as baixas coberturas vacinais. No município de Tubarão/SC, a cobertura vacinal para o influenza em 2006 foi de $83,7 \%{ }^{16}$. Em um estudo realizado em Teresina/PI, com o objetivo de levantar os conhecimentos, atitudes e práticas dos idosos sobre a vacina, bem como, identificar os motivos que levaram alguns a não se vacinarem verificou-se que mesmo com o alcance das coberturas vacinais muitos idosos continuam acreditando que a vacina, ao invés de oferecer proteção, oferece riscos, gerando resistência e trazendo dificuldades à execução das campanhas ${ }^{4}$. Desta maneira, como aponta Donalísio cols ${ }^{15}$, os estudos sobre a percepção e apresentação dos sintomas após a vacinação podem esclarecer parte dos motivos de baixas coberturas vacinais e contribuir para intervenções educativas mais específicas.

Em vista disso, este trabalho buscou investigar a incidência de efeitos adversos após a vacinação contra influenza, nos vacinados na rede púbica no município de Tubarão, na Campanha Nacional de Vacinação do Idoso de 2008. Ainda, foi objetivo deste trabalho verificar o efeito protetor da vacina contra influenza, comparando os indivíduos vacinados pela rede pública com os não vacinados.

\section{MÉTODOS}

Foi realizado um estudo de coorte prospectivo, durante os meses de abril a setembro de 2008, em idosos com idade igual ou superior a 60 anos, residentes em Tubarão. A amostra do estudo foi composta por dois grupos. O primeiro grupo foi formado por aqueles que receberam a dose da vacina contra influenza nos postos de saúde do SUS, o segundo, por aqueles que, por escolha própria, não foram vacinados. Este último grupo foi selecionado na farmácia central do município ou em serviços de extensão da universidade e da prefeitura.

O recrutamento dos participantes ocorreu nos meses de abril e maio de 2008. Os critérios de inclusão foram: ter idade igual ou superior a 60 anos e aceitar participar do estudo. Foram excluídos do estudo aqueles que tinham sido recrutados para o grupo de não vacinados, mas receberam a vacina posteriormente. Optou-se por não excluir os indivíduos que tomaram outras vacinas na mesma ocasião, embora tal situação pudesse alterar a análise de eventos adversos.

Os participantes do estudo foram entrevistados no momento do recrutamento, sendo aplicado um questionário contendo perguntas sobre: sexo, escolaridade, idade, tabagismo, índice de massa corporal, risco para doenças cardiovasculares, presença de hipertensão arterial, presença de diabetes mellitus, presença de doença pulmonar obstrutiva crônica e presença de alergia. Também foram verificadas características quanto à vacinação em algum ano anterior, o número médio estimado de resfriado por ano, e se ao fazer a vacina contra influenza também recebeu outra vacina.

Após o recrutamento, os participantes recebiam uma ficha para o automonitoramento e preenchimento domiciliar. Esta ficha era dividida em duas partes. A primeira relacionada com os efeitos adversos nos primeiros 15 dias e a segunda com os sinais e sintomas gripais para todo o período de acompanhamento, que foi até o mês de setembro, correspondente ao início da primavera.

Após um período de sete a quinze dias da vacinação, os indivíduos vacinados foram contatados por telefone e questionados sobre reações locais como dor, vermelhidão e endurecimento, e quanto a manifestações sistêmicas como febre, mal estar, dor muscular, dor de cabeça, íngua ou caroço, diarréia, vômito, secreção nasal, dor no peito, tosse, dor de ouvido, dor nas articulações, alergia e outros sintomas. Para aqueles que relataram algum efeito foi realizado uma visita domiciliar por um dos integrantes da pesquisa para a confirmação dos dados.

A ficha de acompanhamento de sinais e sintomas gripais continha dados referentes à presença, no período, de: febre, mal estar, dor muscular, calafrios, dor de cabeça, secreção nasal, dor de garganta, tosse, dor no peito, dor ao movimentar os olhos. Para cada item afirmativo o sujeito da pesquisa deveria responder a data de início da apresentação, tempo de duração e se fez uso de alguma medicação. Mensalmente, foram realizados contatos telefônicos com cada um dos participantes e, nos casos em que os mesmos relatavam a presença de algum destes sintomas, foi realizada a visita domiciliar por um dos autores. A presença de febre foi orientada como temperatura corporal acima de $38^{\circ} \mathrm{C}$.

A presença de efeitos adversos foi verificada através do autorrelato do paciente e classificada como reação local e reações sistêmicas. $\mathrm{O}$ efeito protetor da vacina foi verificado através do autorrelato do paciente em relação à presença, durante os meses de inverno, dos sintomas descritos anteriormente e comumente presentes na síndrome gripal.

Os dados foram analisados pelo programa InStat3 através de frequência simples e a associação foi avaliada através do risco relativo com um nível de significância de $5 \%$. As análises de comparação entre os grupos foi realizada por tabelas de contingência $2 \times 2$ e pelo teste exato de Fisher.

\section{Considerações éticas}

O estudo foi aprovado pelo Comitê de Ética de Pesquisa da Universidade do Sul do Estado de Santa Catarina sob registro 08.077.4.01. III.

\section{RESULTADOS}

Foram recrutados inicialmente 351 indivíduos, porém, no decorrer do estudo houve perda de 10 participantes (dois óbitos, oito não localizados). Desta maneira, a amostra foi de 341 pacientes, sendo 289 vacinados pela rede pública (VSUS) e 52 não vacinados (NV).

As características sócio-culturais e clínicas são apresentadas na Tabela 1, enquanto os antecedentes vacinais dos participantes do estudo são apresentados na Tabela 2. A maioria dos indivíduos do grupo VSUS relatou haver recebido a vacina contra influenza em anos anteriores (Tabela 2).

Em relação às características clínicas, homens que apresentaram uma circunferência da cintura maior que $94 \mathrm{~cm}^{17}$, caracterizados homens com um risco maior de complicações cardiovasculares, procuram mais pela vacina do que aqueles que não possuíam esse risco $(p=0,0059)$ (Tabela 1 ).

Segundo relatos dos participantes, a média do número de resfriados que os acometem por ano é de 2,079 no grupo VSUS (mediana $=2$; desvio padrão $=1,829)$; e de 1,545 no grupo NV $($ mediana $=1$; desvio padrão $=1,011)$ (Tabela 2).

Nossos dados mostram que cerca de $15 \%$ da população que tomou a vacina em 2008 não se vacinou no ano anterior, enquanto $30 \%$ da população que recebeu a vacina no ano anterior não se vacinou no ano do estudo (Tabela 2). 
TABELA 1 - Características sócioculturais e clínicas dos participantes.

\begin{tabular}{|c|c|c|c|c|}
\hline \multirow[b]{2}{*}{ Características } & \multicolumn{2}{|c|}{$\operatorname{VSUS}\left(\mathrm{n}^{\mathrm{o}}=289\right)$} & \multicolumn{2}{|c|}{$\mathrm{NV}\left(\mathrm{n}^{\mathrm{o}}=52\right)$} \\
\hline & $\mathrm{n}^{\mathrm{o}}$ & $\%$ & $\mathrm{n}^{\mathrm{o}}$ & $\%$ \\
\hline \multicolumn{5}{|l|}{ Sexo } \\
\hline masculino & 114 & 39,4 & 11 & 21,2 \\
\hline feminino & 175 & 60,6 & 41 & 78,8 \\
\hline \multicolumn{5}{|l|}{ Escolaridade } \\
\hline analfabeto & 11 & 3,8 & 0 & - \\
\hline até $8^{\mathrm{a}}$ série & 201 & 69,6 & 37 & 71,1 \\
\hline segundo grau completo & 38 & 13,2 & 5 & 9,6 \\
\hline superior completo & 19 & 6,6 & 3 & 5,8 \\
\hline não informou & 18 & 6,2 & 7 & 13,5 \\
\hline complementar & 1 & 0,3 & 0 & - \\
\hline normalista & 1 & 0,3 & 0 & - \\
\hline \multicolumn{5}{|l|}{ Faixa etária } \\
\hline $60-65$ & 99 & 34,3 & 25 & 48,1 \\
\hline $65-70$ & 71 & 24,6 & 12 & 23,1 \\
\hline $70-75$ & 63 & 21,8 & 7 & 13,5 \\
\hline $75-80$ & 36 & 12,4 & 6 & 11,5 \\
\hline $80-85$ & 13 & 4,4 & 2 & 3,8 \\
\hline $85-90$ & 4 & 1,4 & 0 & - \\
\hline$>90$ & 3 & 1,0 & 0 & - \\
\hline \multicolumn{5}{|l|}{ Tabagista } \\
\hline $\operatorname{sim}$ & 18 & 6,2 & 4 & 7,7 \\
\hline não & 271 & 93,8 & 48 & 92,3 \\
\hline
\end{tabular}

\section{Índice de massa corporal}

$\begin{array}{lrrrr}\text { baixo peso } & 3 & 1,0 & 0 & 0,0 \\ \text { peso normal } & 108 & 37,4 & 24 & 46,2 \\ \text { sobre peso } & 114 & 39,5 & 17 & 32,7 \\ \text { obesidade } & 59 & 20,4 & 10 & 19,2 \\ \text { não informou } & 5 & 1,7 & 1 & 1,9\end{array}$

\begin{tabular}{lrrrr}
\hline Risco para doenças cardiovasculares & & & & \\
mulheres com risco aumentado & 141 & 48,8 & 29 & 55,8 \\
mulheres sem risco & 13 & 4,5 & 1 & 1,9 \\
não informou & 21 & 7,3 & 11 & 21,1 \\
homens com risco aumentado & 70 & 24,2 & 4 & 7,7 \\
homens sem risco & 33 & 11,4 & 3 & 5,8 \\
não informou & 11 & 3,8 & 4 & 7,7 \\
\hline Hipertensão artéria sistêmica & & & & \\
sim & 187 & 64,7 & 32 & 61,5 \\
não & 102 & 35,3 & 20 & 38,5 \\
\hline Diabetes mellitus & & & & \\
sim & 54 & 18,7 & 9 & 17,3 \\
não & 235 & 81,3 & 43 & 82,7 \\
\hline Doença pulmonar obstrutiva crônica & & & & \\
sim & 17 & 5,9 & 2 & 3,8 \\
não & 272 & 94,1 & 50 & 96,2 \\
\hline Alergia & & & & \\
sim & 55 & 19,0 & 10 & 19,2 \\
não & 234 & 81,0 & 42 & 80,8 \\
\hline
\end{tabular}

VSUS: vacinados na rede pública, NV: não vacinados.

Ao investigar a incidência dos efeitos adversos no grupo VSUS verificou-se um percentual de $22,5 \%$ da presença de pelo menos um efeito adverso. Os efeitos adversos referidos pelos pacientes mais frequentes foram mal estar $(11,8 \%)$, secreção nasal $(8,7 \%)$ e dor de cabeça $(7,6 \%)$ (Tabela 3).
TABELA 2 - Características vacinais dos participantes.

\begin{tabular}{|c|c|c|c|c|}
\hline \multirow[b]{2}{*}{ Características } & \multicolumn{2}{|c|}{ VSUS $\left(\mathrm{n}^{\circ}=289\right)$} & \multicolumn{2}{|c|}{$\mathrm{NV}\left(\mathrm{n}^{\circ}=52\right)$} \\
\hline & $\mathrm{n}^{\mathrm{o}}$ & $\%$ & $\mathrm{n}^{\mathrm{o}}$ & $\%$ \\
\hline \multicolumn{5}{|c|}{ Vacinou-se anteriormente } \\
\hline $\operatorname{sim}$ & 246 & 85,1 & 15 & 28,8 \\
\hline não & 43 & 14,9 & 37 & 71,2 \\
\hline \multicolumn{5}{|c|}{ Vacinou-se ano passado } \\
\hline $\operatorname{sim}$ & 243 & 84,0 & 15 & 28,8 \\
\hline não & 46 & 16,0 & 37 & 71,2 \\
\hline \multicolumn{5}{|c|}{ Número médio de resfriados por ano } \\
\hline $1 x$ ou $2 x$ & 121 & 41,9 & 19 & 36,5 \\
\hline $3 \mathrm{x}$ ou mais & 31 & 10,7 & 3 & 5,8 \\
\hline não informou & 137 & 47,4 & 30 & 57,7 \\
\hline \multicolumn{5}{|c|}{ Recebeu outra vacina } \\
\hline $\operatorname{sim}$ & 14 & 4,8 & - & - \\
\hline não & 275 & 95,2 & - & - \\
\hline
\end{tabular}

VSUS: vacinados na rede pública, NV: não vacinados.

TABELA 3 - Incidência dos efeitos adversos após a vacina nos idosos vacinados na rede pública.

\begin{tabular}{|c|c|c|c|c|}
\hline \multirow[b]{2}{*}{ Características } & \multicolumn{2}{|c|}{ VSUS $\left(n^{\circ}=289\right)$} & \multirow[b]{2}{*}{ RR (IC 95\%) } & \multirow[b]{2}{*}{ P valor } \\
\hline & $\mathrm{n}^{\mathrm{o}}$ & $\%$ & & \\
\hline Qualquer sintoma* & 65 & 22,5 & $1,00(0,96-1,06)$ & 1,0000 \\
\hline \multicolumn{5}{|l|}{ Reação local } \\
\hline dor & 11 & 3,8 & - & - \\
\hline hiperemia & 2 & 0,7 & - & - \\
\hline endurecimento & 3 & 1,0 & - & - \\
\hline \multicolumn{5}{|l|}{ Reação sistêmica } \\
\hline febre & 20 & 6,9 & $0,98(0,89-1,09)$ & 0,5562 \\
\hline mal estar & 34 & 11,8 & $0,97(0,90-1,06)$ & 0,6276 \\
\hline dor muscular & 16 & 5,5 & $0,92(0,78-1,08)$ & 0,1356 \\
\hline cor de cabeça & 22 & 7,6 & $0,99(0,90-1,09)$ & 0,5906 \\
\hline linfonodomegalia & 1 & 0,3 & - & - \\
\hline diarréia & 3 & 1,0 & $0,78(0,44-1,37)$ & 0,1395 \\
\hline vômito & 4 & 1,4 & - & - \\
\hline secreção nasal & 25 & 8,7 & $1,00(0,92-1,08)$ & 1,0000 \\
\hline dor no peito & 10 & 3,5 & - & - \\
\hline tosse & 16 & 5,5 & $0,92(0,78-1,08)$ & 0,1356 \\
\hline dor de ouvido & 2 & 0,7 & - & - \\
\hline dor nas articulações & 8 & 2,8 & - & - \\
\hline alergia & ** & ** & - & - \\
\hline outro & 11 & 3,8 & - & - \\
\hline
\end{tabular}

RR: risco relativo, IC: intervalo de confiança. ${ }^{*}$ Indivíduos que referiram pelo menos um efeito adverso após a vacina. VSUS: vacinados na rede pública, ${ }^{* *}$ sintoma não relatado no grupo.

Embora tenham sido testadas, não foram encontradas associações significativas entre a ocorrência de qualquer efeito adverso e a presença de hipertensão arterial, diabetes mellitus, doença pulmonar obstrutiva crônica ou alergia (Tabela 1).

A Tabela 4 mostra a incidência dos sintomas gripais no grupo VSUS e NV, sendo a tosse o sintoma mais relatado por todos os pacientes. Houve diferença significativa entre os grupos em relação às variáveis tosse e dor de garganta $(\mathrm{p}=0,0225$ e $\mathrm{p}=0,0407$, respectivamente). Em relação à febre, houve uma tendência a maior incidência do sintoma no grupo vacinado do que no grupo não vacinado $(p=0,0553)$. Comparando estes grupos quanto à presença 
TABELA 4 - Incidência de sinais e sintomas gripais após vacinação em idosos vacinados na rede pública ou não vacinados.

\begin{tabular}{|c|c|c|c|c|c|c|}
\hline \multirow[b]{2}{*}{ Característica } & \multicolumn{2}{|c|}{$\begin{array}{c}\text { VSUS } \\
\left(\mathrm{n}^{\mathrm{o}}=289\right)\end{array}$} & \multicolumn{2}{|c|}{$\begin{array}{c}\mathrm{NV} \\
(\mathrm{n}=52)\end{array}$} & \multirow[b]{2}{*}{ RR (IC 95\%) } & \multirow[b]{2}{*}{ p valo } \\
\hline & $\overline{\mathrm{n}^{\mathrm{o}}}$ & $\%$ & $\overline{\mathrm{n}^{\mathrm{o}}}$ & $\%$ & & \\
\hline Febre & 46 & 17,6 & 3 & 5,8 & $1,12(1,03-1,23)$ & 0,0553 \\
\hline Mal estar & 82 & 28,4 & 13 & 25,0 & $1,02(0,93-1,13)$ & 0,7373 \\
\hline Dor muscular & 56 & 19,4 & 8 & 15,4 & $1,04(0,93-1,15)$ & 0,5678 \\
\hline Calafrio & 37 & 12,8 & 2 & 3,8 & $1,13(1,04-1,24)$ & 0,0935 \\
\hline Dor de cabeça & 56 & 19,4 & 7 & 13,5 & $1,06(0,95-1,17)$ & 0,4369 \\
\hline Secreção nasal & 90 & 31,1 & 13 & 25,0 & $1,04(0,95-1,14)$ & 0,4160 \\
\hline Dor de garganta & 42 & 14,5 & 2 & 3,8 & $1,14(1,05-1,24)$ & 0,0407 \\
\hline Tosse & 96 & 33,2 & 9 & 17,3 & $1,11(1,02-1,21)$ & 0,0225 \\
\hline Dor no peito & 24 & 8,3 & 1 & 1,9 & $1,14(1,04-1,25)$ & 0,1470 \\
\hline Dor ao movimentar os olhos & 7 & 2,4 & * & - & - & \\
\hline Outros & 10 & 3,5 & 2 & 3,8 & $0,98(0,75-1,27)$ & 1,0000 \\
\hline Qualquer sintoma** & 136 & 47,0 & 15 & 28,8 & $1,11(1,02-1,22)$ & 0,0156 \\
\hline
\end{tabular}

de pelo menos um sintoma gripal, foi verificada uma incidência de $47 \%$ e $28,8 \%(R R=1,11 ; 1.02-1.22 ; \mathrm{p}=0,0156)$, respectivamente. A presença associada de tosse e febre foi encontrada numa frequência de $12,8 \%$ e $1,9 \%(\mathrm{RR}=6.65 ; 0.93-47.48 ; \mathrm{p}=0.0165)$, nos grupos VSUS e NV, respectivamente.

\section{DISCUSSÃo}

$\mathrm{Na}$ amostra estudada, para todos os grupos, observou-se que a proporção de mulheres em relação aos homens incluídas no estudo é maior (Tabela 1). Isso pode ser explicado, pois a proporção de mulheres acima de 60 anos é sabidamente superior a masculina nesta mesma faixa etária. Ainda, as mulheres possuem um maior conhecimento sobre as doenças e, desta maneira, são mais atentas ao aparecimento de sinais e sintomas, além de procurarem mais os serviços de saúde do que os homens ${ }^{18,19}$.

No grupo investigado, verificou-se a predominância de idosos na faixa etária entre 65 e 75 anos (46,3\%), o que vem ao encontro dos resultados de outros estudos semelhantes, os quais afirmam que a população brasileira está atingindo vida média acima de $65 \operatorname{anos}^{20} \mathrm{e}$ de que a probabilidade de ser vacinado aumenta com a idade ${ }^{21-23}$.

Sabe-se que a vacinação contra a infecção pelo vírus da influenza é formalmente indicada para pessoas em risco de complicações em caso de infecção, como os adultos e crianças com doenças pulmonares ou cardiovasculares crônicas e com doenças metabólicas crônicas ${ }^{1}$. A maior parte dos idosos vacinados, com exceção dos portadores de doença pulmonar, referiu ser portadora de doenças crônicas como diabetes mellitus e hipertensão arterial (Tabela 1). Além disso, o aumento da gordura corporal está associado ao aumento de prevalência de fatores de risco para doenças cardiovasculares, como dislipidemia e intolerância à glicose e principalmente sobre a prevalência de hipertensão arterial ${ }^{24}$. No estudo, esses pacientes demonstraram uma procura significativa ao serviço de saúde (Tabela 1).

Segundo Donalísio cols ${ }^{25}$, que obtiveram o mesmo resultado que o presente estudo, uma hipótese explicativa seria que a maior proximidade desses pacientes com os serviços de saúde públicos facilite o seu comparecimento às campanhas. Por outro lado,
Moura \& Silva ${ }^{26}$, observaram que profissionais de saúde indicam pouco a vacina, mesmo para pacientes que teriam indicação formal.

Em relação ao nosso achado de que $30 \%$ da população que recebeu a vacina no ano anterior não se vacinaram no ano do estudo, isso pode estar relacionado com conceitos errôneos da população em relação aos riscos e benefícios da vacina contra a influenza. Uma pesquisa realizada em uma área do Programa Saúde da Família (PSF), em Terezina/PI, com idosos na faixa etária a partir de 60 anos, observou um conhecimento inadequado, com conceitos desvinculados dos objetivos reais da vacina para a saúde do idoso, verificando-se inseguranças referentes à suas indicações, contraindicações e, principalmente, em relação aos efeitos adversos ${ }^{4}$. Esse, como já comentado neste estudo, vem sendo apontado como fator que contribui para as baixas coberturas vacinais ${ }^{15,27}$.

Em relação aos efeitos adversos, embora tenha sido observado $22,5 \%$ de indivíduos com relato de pelo menos um efeito, estes não foram sintomas graves, sugerindo pouca reatogenicidade da vacina, corroborando com resultados de outros autores ${ }^{10,6}$.

Quanto ao tipo de efeito adverso apresentado pelos participantes, entre as reações locais, a dor foi o mais frequente e, entre as reações sistêmicas, o mal estar. Mesmo assim, foi um grupo pequeno de indivíduos que relataram estas queixas. Outro estudo também aponta o mal estar como a reação sistêmica mais frequente ${ }^{27}$.

Em nosso trabalho, apresentação de sinais e sintomas respiratórios durante o inverno de 2008 foi maior no VSUS comparado ao NV, embora só tenhamos encontrado significância estatística entre os sintomas dor de garganta e tosse (Tabela 4). Ainda, o grupo vacinado apresentou seis vezes mais chance de apresentar tosse e febre, indicando que o grupo VSUS teria maior incidência de sinais gripais que o NV.

No entanto, considerando que o quadro clínico característico da gripe é composto não apenas de um sintoma isolado, mas sim pelo conjunto: febre, calafrios, cefaléia, tosse seca, dor de garganta, congestão nasal ou coriza, mialgia, anorexia e fadiga ${ }^{28,29}$, foi realizada análise da incidência dos sintomas associados. Quando feita esta associação com os dados obtidos, observamos que ela está presente em $1,3 \%$ dos pacientes VSUS comparado ao NV, não sendo essa uma associação significante (Tabela 4).

Tais resultados podem estar relacionados com o perfil dos pacientes que costumam tomar a vacina. Parece que, em geral, indivíduos mais suscetíveis ao vírus influenza são os que buscam a imunoproteção da vacina. Isto, considerando que os pacientes que procuram a vacinação têm como objetivo principal se prevenir contra quadros graves, que possam levá-los à internação hospitalar. Tal comportamento é congruente com a orientação de recomendações da vacinação para influenza, cuja intenção é a prevenção da gripe em populações de alto risco para a doença severa e/ou complicações, e de fato, tais situações não foram significativas entre os participantes do estudo.

A investigação apresentou algumas limitações. Primeiro, a presença de viés de aferição, uma vez que indivíduos de baixa escolaridade podem ter tido dificuldade no preenchimento dos formulários, ou também pelo fato do acompanhamento ter sido realizado por telefone, ou ainda, que somente eram visitados pelos pesquisadores aqueles indivíduos que apresentassem sintomas. Segundo, o estudo utilizou como desfecho para a verificação do efeito protetor da vacina a presença de sinais e sintomas gripais. Não foram avaliados desfechos de maior relevância clínica como pneumonia, internação hospitalar ou morte. 
É também digno de nota que ao se investigar doenças respiratórias, direta ou indiretamente associadas a infecções pelo vírus influenza, deve-se considerar variáveis como a circulação de outros vírus respiratórios de importância clínica e epidemiológica, circulação de bactérias e outros patógenos ${ }^{10}$. Deve-se também considerar que a vacinação não previne a população contra qualquer infecção de vírus influenza, mas somente os casos de infecção pelas linhagens virais que fazem parte da composição da vacina na estação ${ }^{30}$.

Esse trabalho mostrou pela primeira vez a incidência de efeitos adversos após a vacinação contra influenza na população idosa do município de Tubarão/SC, na rede pública e comparou o efeito protetor da vacina com indivíduos não vacinados.

Em conclusão, a vacina contra o influenza pode ser considerada pouco reatogênica, visto que os efeitos adversos pós-vacinais apresentados foram pouco incidentes e sem gravidade.

Embora idosos vacinados tenham se mostrado mais susceptíveis à apresentação de sintomas gripais do que os não vacinados, essas manifestações não tiveram grande relevância clínica e pode estar relacionado ao perfil do paciente que faz a vacina.

\section{AGRADECIMENTOS}

Agradecemos o apoio da Secretaria Municipal de Saúde de Tubarão e da Clínica Pró-Vida de Tubarão. Agradecemos aos graduandos do Curso de Medicina da Universidade do Sul de Santa Catarina (UNISUL) pela ajuda no recrutamento dos participantes.

\section{CONFLITO DE INTERESSE}

Os autores declaram não haver nenhum tipo de conflito de interesse no desenvolvimento do estudo.

\section{SUPORTE FINANCEIRO}

Programa Prêmio Mérito Universitário da Fundação de Apoio à Pesquisa Científica e Tecnológica do Estado de Santa Catarina (FAPESC) e Universidade do Sul de Santa Catarina (UNISUL).

\section{REFERÊNCIAS}

1. Neto EF, Halker E, Santos VJ, Palva TM, Neto JT. Influenza. Rev Soc Bras Med Trop 2003; 36:267-274

2. Nichol KL, Margolis KL, Wuorenma J, Von Sternberg T. The efficacy and cost effectiveness of vaccination against influenza among elderly persons in the community. N Engl J Med 1994; 331:778-784.

3. Ministério da Saúde. Manual de Vigilância epidemiológica dos eventos adversos pós-vacinação. Brasília: Ministério da Saúde; 1998.

4. Araújo TME, Lino FS, Nascimento DJC, Costa FSR. Vacina contra Influenza: conhecimentos, atitudes e práticas de idosos em Teresina. Rev Bras Enferm 2007; 60:439-443.

5. Centers for Disease Control and Prevention. Prevention and Control of Influenza: recommendations of the Advisory Committee on Immunization Practices. MMWR Morb Mortal Wkly Rep 2000; 49(RR03):1-38.

6. Francisco PMSB, Donalísio MRC, Lattorre RDO. Impacto da vacinação contra influenza na mortalidade por doenças respiratórias em idosos. Rev Saude Publica 2005; 39:75-81.

7. Gross PA, Hermogenes AW, Sacks HS, Lau J, Levandowski RA. The efficacy of influenza vaccine in elderly persons: a meta-analysis and review of the literature. Ann Intern Med 1995; 123:518-527.
8. Kaiser L, Couch RB, Galasso GJ, Gleznen WP, Webster RG, Wright PF, et al. First international symposium on influenza and other respiratory viruses: summary and overview. Antiviral Res 1999; 42:149-175.

9. Ohmit SE, Monto AS. Influenza vaccine effectiveness in preventing hospitalization among the elderly during influenza type A and type B seasons. Int J Epidemiol 1995 ; $24: 1240-1248$.

10. Donalísio MR, Francisco PMSB, Latorre MRDO. Tendência da mortalidade por doenças respiratórias em idosos antes e depois das campanhas de vacinação contra influenza no Estado de São Paulo - 1980 a 2004. Rev Bras Epidemiol 2006; 9:32-41.

11. Façanha MC. Impacto da Vacinação de maiores de 60 anos para influenza sobre as internações e óbitos por doenças respiratórias e circulatórias em Fortaleza CE - Brasil. J Bras Pneumol 2005; 31:415-420.

12. Ramadan PA, Araújo FB, Ferreira Junior M. A 12-month follow-up of an influenza vaccination campaign based on voluntary adherence: report on upper-respiratory symptoms among volunteers and non-volunteers. Sao Paulo Med J 2001; 119:142-145.

13. Toniolo Neto J, Weckx LW, Halker E, Lopes CH, Succi RCM, Paiva TM, et al. Safety of simultaneous pneumococcal and influenza vaccination in elderly patients in Brazil. Drugs Aging 1999; 15:43-45.

14. Weckx LY,Lopes $\mathrm{CH}$, Kobayashi FK. Avaliação da tolerância à vacina contra influenza e à vacina contra pneumococo em idosos. In: Boletim Informativo. São Paulo: Centro de Vigilância Epidemiológica. Secretaria Estadual de Saúde; 1999. p.14:7-10.

15. Donalísio MR, Ramalheira RM, Cordeiro R. Eventos adversos após vacinação contra influenza em idosos, Distrito de Campinas, SP, 2000. Rev Soc Bras Med Tropical 2003; 36:467-471.

16. Banco de dados do Sistema Único de Saúde [Internet]. Cadernos de Informações de Saúde - Município: Tubarão - SC. [Acessado 21 nov 2007]. Disponível em: http://tabnet.datasus.gov.br/tabdata/cadernos/SC/SC Tubarão Geral. XLS/.

17. World Health Organization. Preventing and managing the global epidemic of obesity. Geneva: Report of the World Health Organization Consultation of Obesity; 1997.

18. Sales FM, Santos I. Perfil dos idosos hospitalizados e nível de dependência de cuidados de enfermagem: identificação de necessidades. Texto Contexto Enferm 2007; 16:495-502.

19. Araujo TCN, Alves MIC [Internet]. Perfil da população idosa no Brasil. Textos Envelhecimento. [Acessado em 12 fev 2009]. Disponível em: http://www.unati.uerj.br/tse/scielo.php?script=sci_arttext\&pid=S1517$59282000000100002 \& \operatorname{lng}=\mathrm{pt} \& \mathrm{nrm}=\mathrm{iso} /$.

20. Donalísio MR, Bergamo PMSF, Latorre DO. Intervenções por doenças respiratórias em idosos e a intervenção vacinal contra influenza no Estado de São Paulo. Rev Bras Epidemiol 2004; 2:5-10.

21. Dannetun E, Tegnell A, Normann B, Garpenholt O, Giesecke J. Influenza vaccine coverage and reasons for non vaccination in sample of people above 65 years of age, in Sweden, 1998-2000. Scand J Infect Dis 2003; 35:389-393.

22. Pregliasco F, Sodano L, Mensi C, Selvaggi MT, Adarno B, D’Argenio P, et al. Influenza vaccination among the elderly in Italy. Bul World Health Organ 1999; 77:127-131.

23. Sarriá A, Tirnoner J. Determinants de la vacunación de la gripe en personas mayors de 65 anos. Rev Esp Salud Publica 2002; 76:17-26.

24. Carneiro G, Faria AN, Filho FFR, Guimarães A, Lerário D, Ferreira SRG, et al. Influência da distribuição da gordura corporal sobre a prevalência de hipertensão arterial e outros fatores de cardiovascular em indivíduos obesos. Rev Assoc Med Bras 2003; 49:306-311.

25. Donalísio MR, Ruiz $\mathrm{T}$, Cordeiro R. Fatores associados à vacinação contra influenza em idosos em município do Sudeste do Brasil. Rev Saude Publica 2006; 40:115-119.

26. Moura M, Silva LI. Pesquisa da opinião sobre as campanhas de vacinação contra a influenza no estado de São Paulo. Bol Epidemiol Paulista 2004; 1:8-10.

27. Geronutti DA, Molina AC, Lima SAM. Vacinação de idosos contra a influenza em um centro de saúde escola do interior do estado de São Paulo. Texto Contexto Enferm 2008; 17:336-341.

28. Cox NJ, Fukuda K. Influenza. Infect Dis Clin North Am 1998;12:27-38.

29. Nicholson KG. Human influenza. In: Nicholson KG, Webster RG, Hay AJ, editors. Textbook of influenza. Oxford, UK: Blackwell Science; 1998. p. 219-264.

30. Centers for Disease Control and Prevention. Prevention and Control of Influenza: recommendations of the Advisory Committee on Immunization Practices. MMWR Morb Mortal Wkly Rep 2004; 53:1-40. 